\title{
Synergistic Effects of Plasticizer and 3D Framework toward High-Performance Solid Polymer Electrolyte for Room-Temperature Solid-State Lithium Batteries
}

Jia Wang ${ }^{a, b}$, Jing Yang ${ }^{b}$, Lin Shen $^{b, c}$, Qingya Guo ${ }^{a, b}$, Hao He ${ }^{a *}$, Xiayin Yao ${ }^{b, c *}$

${ }^{\text {a }}$ Key Laboratory of Materials Physics of Ministry of Education, School of Physics and Microelectronics, Zhengzhou University, Zhengzhou, Henan 450001, P. R. China

b Ningbo Institute of Materials Technology and Engineering, Chinese Academy of Sciences, Ningbo 315201, P.R. China

${ }^{c}$ Center of Materials Science and Optoelectronics Engineering, University of Chinese Academy of Sciences, Beijing 100049, P. R.China

Jia Wang and Jing Yang contributed equally to this work.

${ }^{*}$ Corresponding authors

Email addresses: yaoxy@nimte.ac.cn (X.Yao); hehao@zzu.edu.cn (H. He) 


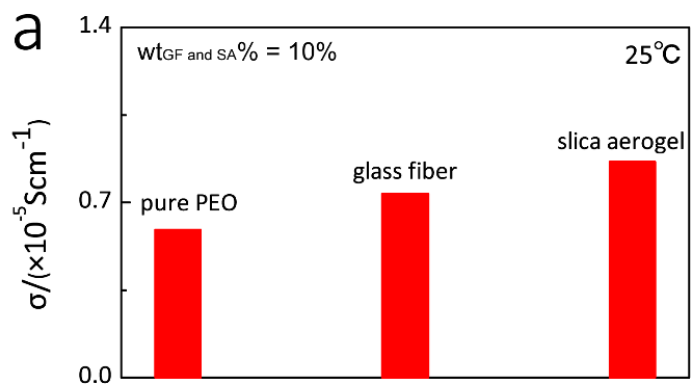

Different material
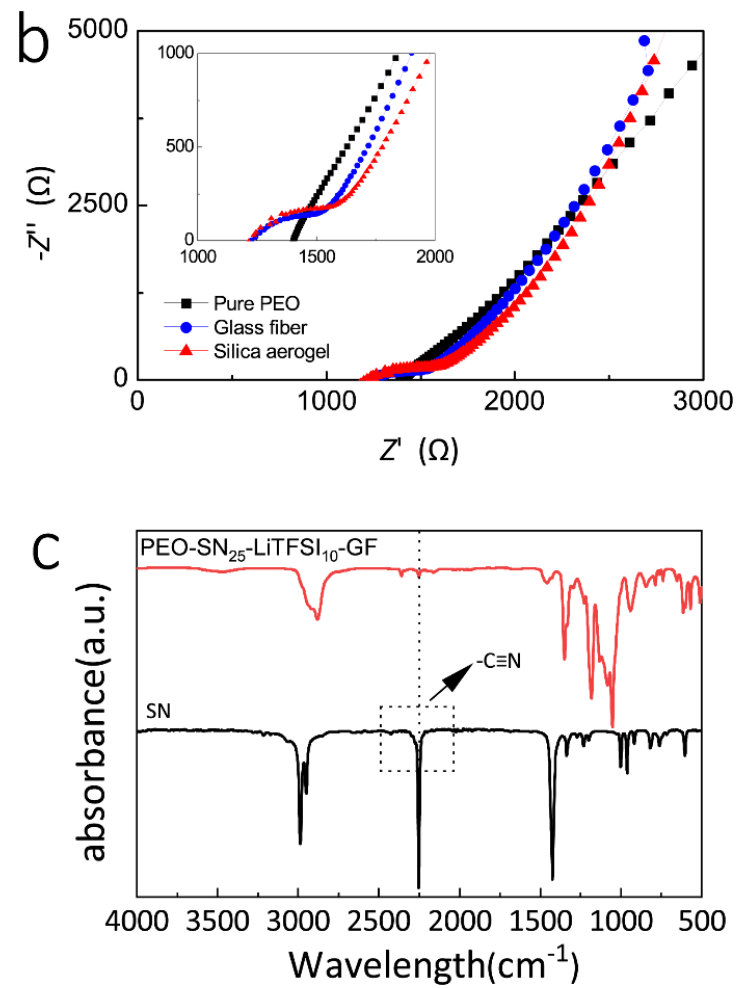

Figure S1 (a) The ionic conductivities of polymer electrolytes with different additives at $25^{\circ} \mathrm{C}$, the weight ratio of GF or SA in electrolyte samples is $10 \mathrm{wt} \%$; (b) Nyquist plot of various PEO-based solid-state electrolytes with different additive at room temperature (EO: $\left.\mathrm{Li}^{+}=18: 1\right)$ (c) FTIR spectra of PEO-SN25-LiTFSI $10-G F$ and SN. 


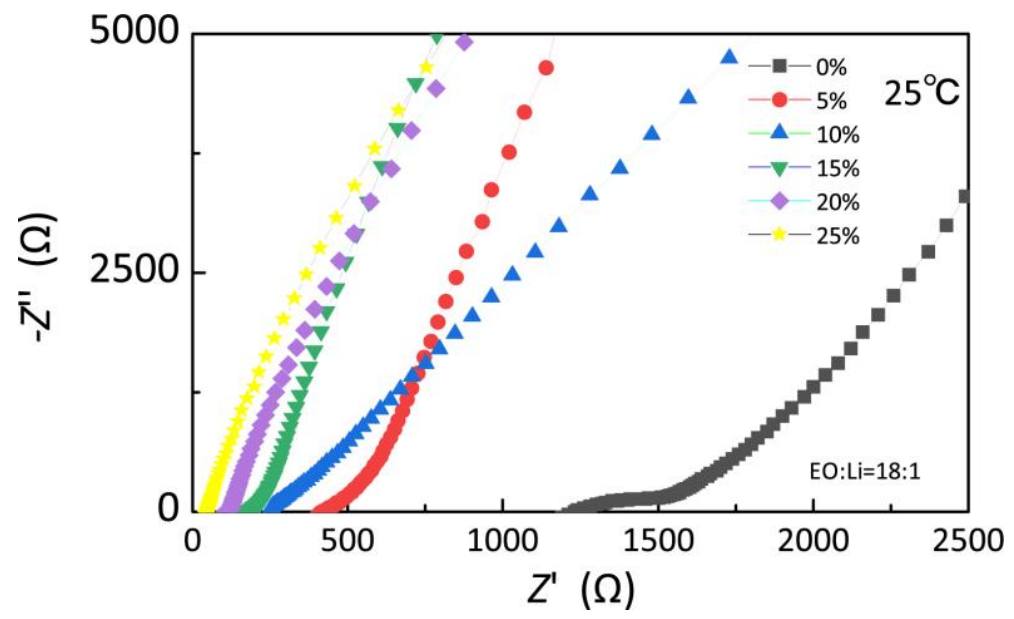

Figure S2 The Nyquist plot of PEO-SN-LiTFSI ${ }_{18}-\mathrm{GF}$ with different ratios of SN and an $\mathrm{EO}: \mathrm{Li}^{+}$ratio of $18: 1$ at $25^{\circ} \mathrm{C}$ 


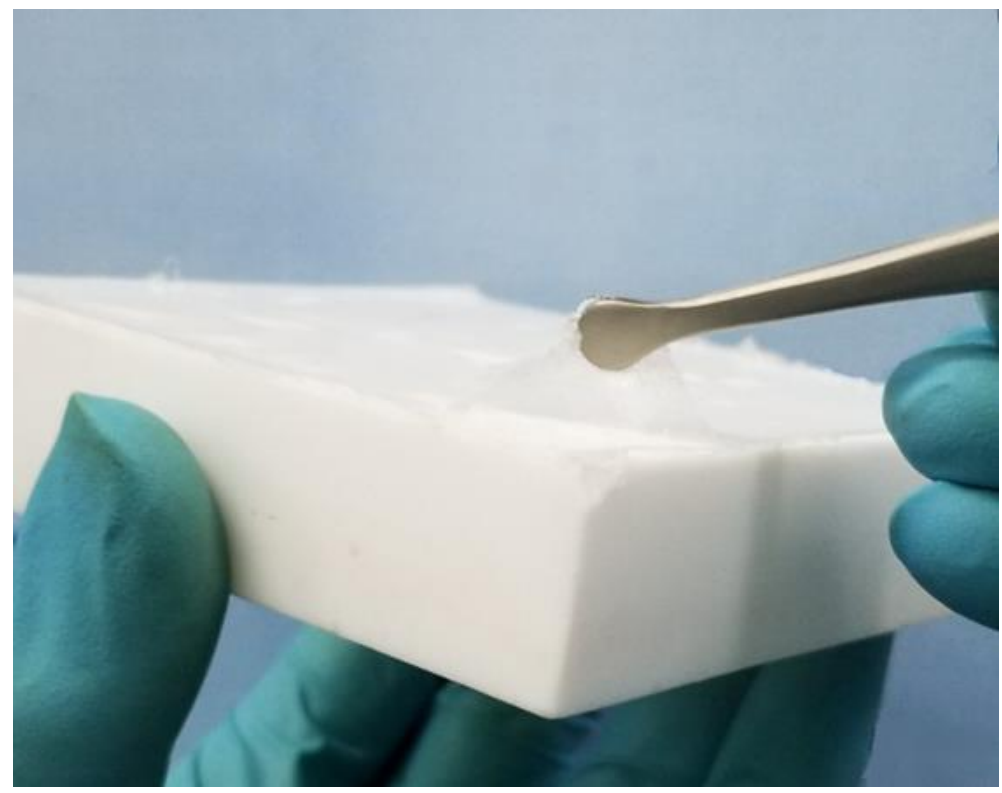

Figure S3 The optical photograph of PEO-SN ${ }_{30}-\mathrm{LiTFSI}_{10}-\mathrm{GF}$ 


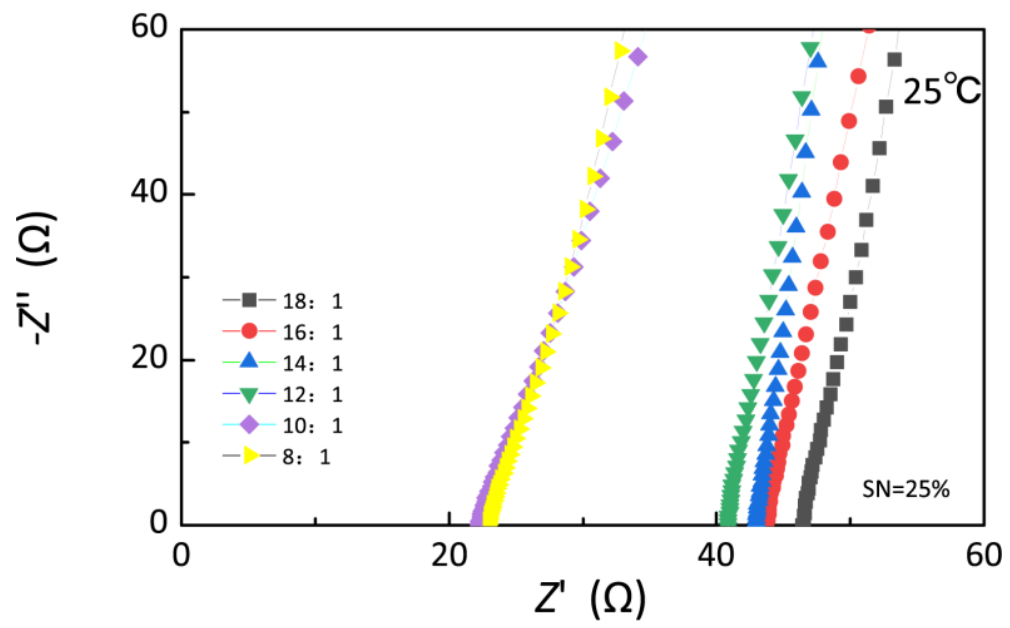

Figure S4 The Nyquist plots of PEO-SN 25 -LiTFSI-GF with different EO: $\mathrm{Li}^{+}$ratios and a SN ratio of $25 \mathrm{wt} \%$ at $25^{\circ} \mathrm{C}$ 

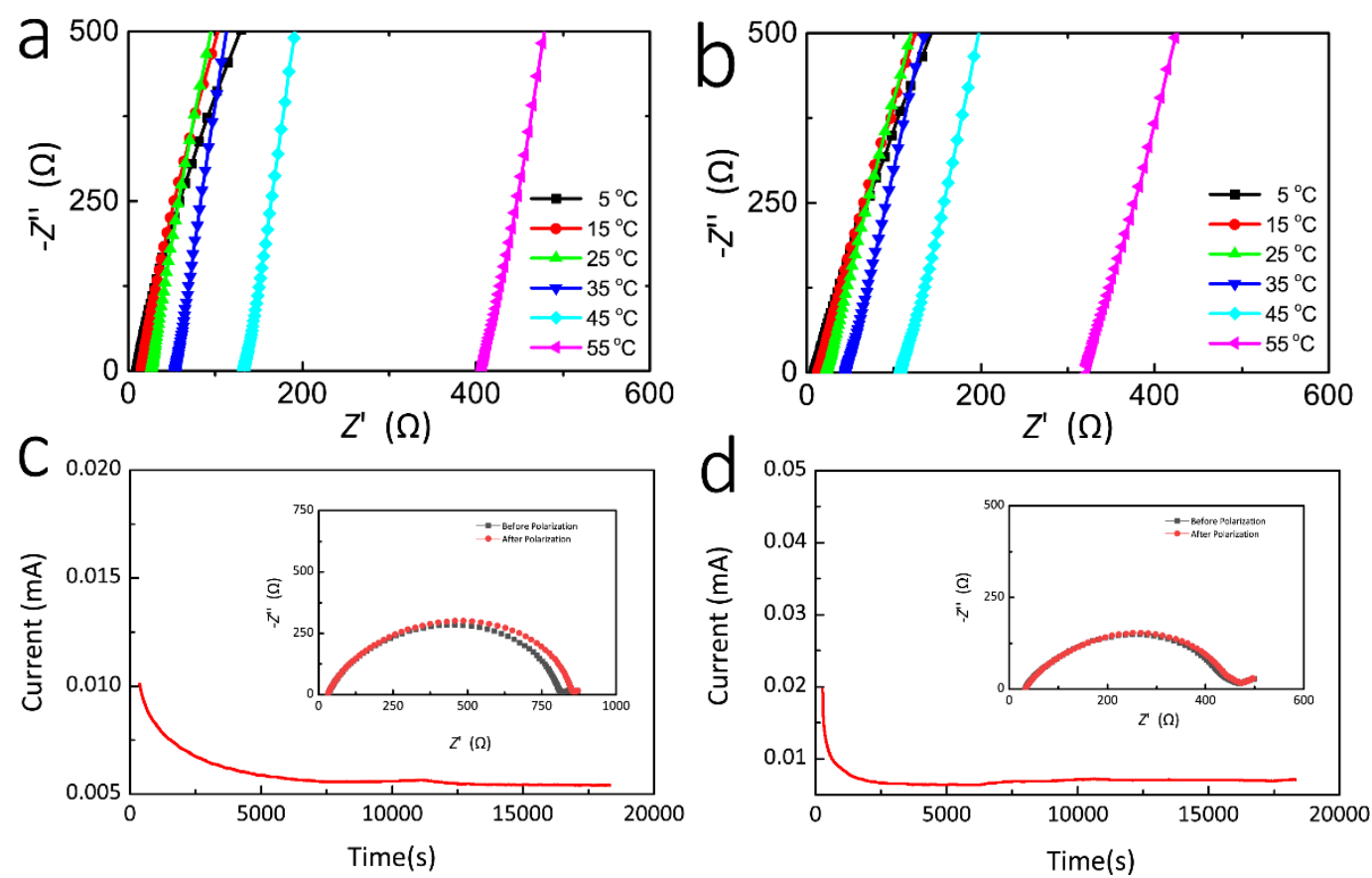

Figure S5 The Nyquist plots of (a) PEO-SN $25-$ LiTFSI $_{10}-\mathrm{GF}$ and (b) PEO-SN 25 LiTFSI $_{10}-\mathrm{SA}$ at different temperatures; (c) Li/PEO-SN ${ }_{25}-\mathrm{LiTFSI}_{10}-\mathrm{SA} / \mathrm{Li}$ and (d) $\mathrm{Li} / \mathrm{PEO}-\mathrm{SN}_{25}$-LiTFSI 10 -GF/Li symmetrical cell under a polarization voltage of $10 \mathrm{mV}$ at room temperature. The corresponding Nyquist plots before and after polarization are shown in the insets. 


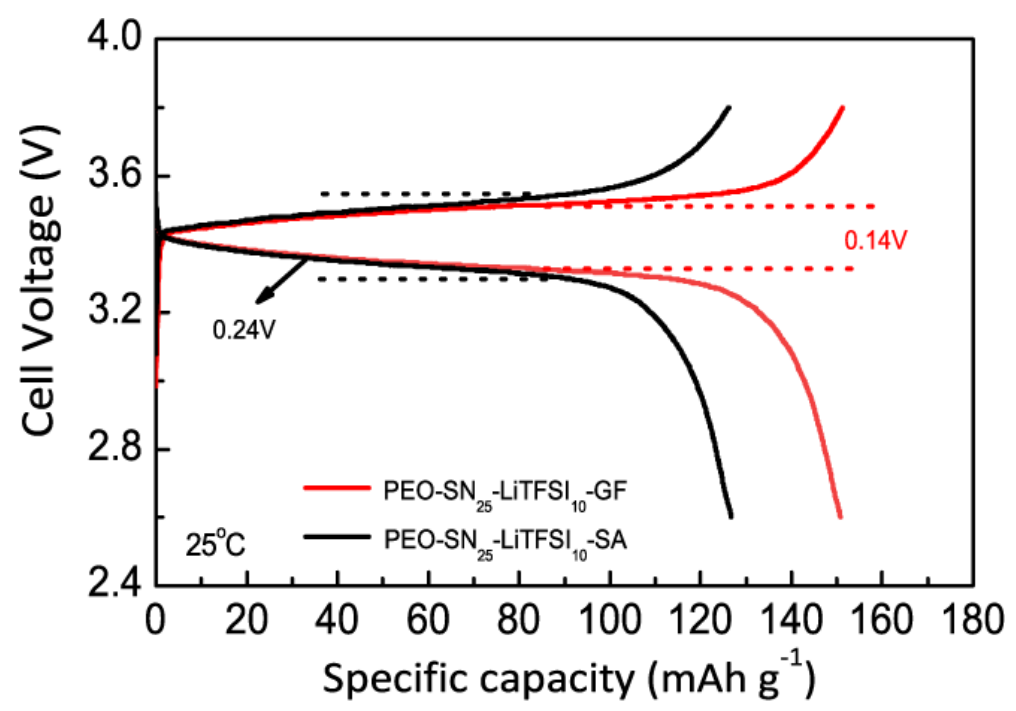

Figure S6 The charge and discharge curves of LFP/PEO-SN ${ }_{25}-\mathrm{LiTFSI}_{10}-\mathrm{GF} / \mathrm{Li}$ and LFP/PEO-SN $25-\mathrm{LiTFSI}_{10}-\mathrm{SA} / \mathrm{Li}$ at $0.1 \mathrm{C}$ under $25^{\circ} \mathrm{C}$. 
Table S1

The relevant data of lithium ion transference numbers $\left(t_{\mathrm{Li}}{ }^{+}\right)$at $25^{\circ} \mathrm{C}$

\begin{tabular}{|c|c|c|c|c|c|c|}
\hline SPEs & $I_{0} / \mu \mathrm{A}$ & $I_{\mathrm{SS}} / \mu \mathrm{A}$ & $R_{0} / \Omega$ & $R_{\mathrm{SS}} / \Omega$ & $\Delta V / \mathrm{mV}$ & $t_{\mathrm{Li}}^{+}$ \\
\hline $\begin{array}{l}\text { PEO (J. Power Sources } \\
2018,387,72-80 \text { ) }\end{array}$ & 178.17 & 41.03 & 25.703 & 29.928 & 10 & 0.14 \\
\hline PEO-SN ${ }_{25}-$ LiTFSI $_{10}$-SA & 10.117 & 5.416 & 787.333 & 829.2 & 10 & 0.20 \\
\hline PEO-SN ${ }_{25}$-LiTFSI ${ }_{10}-\mathrm{GF}$ & 12.296 & 7.1031 & 435.44 & 439.859 & 10 & 0.39 \\
\hline
\end{tabular}




\section{Table S2}

The relevant data of DSC or TGA test results of the prepared SPEs.

\begin{tabular}{lcclc}
\hline Samples & $T_{\mathrm{g}}{ }^{\circ} \mathrm{C}$ & $T_{\mathrm{m}} /{ }^{\circ} \mathrm{C}$ & $\Delta H_{\mathrm{m}} / \mathrm{J} \mathrm{g}$ & $\chi_{\mathrm{c}} / \%$ \\
\hline $\begin{array}{l}\text { PEO (J. Power Sources } \\
\text { 2016, 331, 322-331) }\end{array}$ & -39.6 & 56.29 & 96.7 & 49.24 \\
PEO-LiTFSI ${ }_{18}$-SN 0 -GF & -49 & 45.2 & 34.51 & 25.46 \\
& & & & \\
PEO-LiTFSI $18-\mathrm{SN}_{10}$-GF & -54.1 & 35.4 & 22.19 & 17.96 \\
PEO-LiTFSI $_{18}$ SN $_{25}$-GF & -55.8 & 24.3 & 5.251 & 4.585 \\
\hline
\end{tabular}




\section{Table S3}

Comparison of electrochemical performances of PEO-based solid-state lithium batteries

\begin{tabular}{llllllll}
\hline $\begin{array}{l}\text { Electrolyte } \\
\text { compositions }\end{array}$ & $\begin{array}{l}\mathrm{Li}^{+}: \\
\text {EO }\end{array}$ & $\begin{array}{l}\text { Specific } \\
\text { capacity } \\
\left(\mathrm{mAh} \mathrm{g}^{-1}\right)\end{array}$ & $\begin{array}{l}\text { Cycle } \\
\text { number }\end{array}$ & $\begin{array}{l}\text { Capacity } \\
\text { retention } \\
(\%)\end{array}$ & $\begin{array}{l}\text { Working } \\
\text { temperature } \\
\left({ }^{\circ} \mathrm{C}\right)\end{array}$ & $\begin{array}{l}\text { Current } \\
\text { rate }\end{array}$ & Reference \\
\hline PEO+3DLLTO & $1: 10$ & 90.0 & 30 & 77.8 & 25 & $0.3 \mathrm{C}$ & 41 \\
PEO+GNS & $1: 10$ & 98.1 & 30 & 97.5 & 40 & $0.05 \mathrm{C}$ & 42 \\
PEO+LLZTO@PDA & $1: 8$ & 142.6 & 50 & 99.0 & 50 & $0.2 \mathrm{C}$ & 43 \\
PEO+MOF & $1: 18$ & 151.0 & 100 & 95.0 & 60 & $0.5 \mathrm{C}$ & 44 \\
PEO+LLZO & $1: 16$ & 162.7 & 120 & 91.7 & 60 & $0.1 \mathrm{C}$ & 45 \\
PEO+SN+LiAlO 2 & $1: 10$ & 141.3 & 25 & 84.9 & 60 & $0.1 \mathrm{C}$ & 46 \\
PEO+HAP & $1: 10$ & 141.0 & 300 & 77.0 & 160 & $4 \mathrm{C}$ & 12 \\
PEO+SN+glass fiber & $1: 10$ & 150.0 & 100 & 98.5 & 25 & $0.2 \mathrm{C}$ & This work \\
\hline
\end{tabular}

